[16] K. D. Glazebrook and D. M. Jones. "Some best possible results for a discounted one armed bandit," Metrika, to be published.

[17] P. R. Kumar and T. I. Seidman. "On the optimal solution of the one-armed bandit adaptive control problem," IEEE Trans. Automar. Contr., vol. AC-26. pp. 1176-1184. 1981.

[18] I. Meilijson and G. Weiss, "Multiple feedback at a single server station," Stoc. Proc. Appl. vol. 5, pp. 195-205, 1977

[19] P. Nash, "Optimal allocation of resources between research projects," Ph.D. dissertation. Cambridge University, Cambridge, England. 1973.

[20] P. Nash and J. C. Gittins, "A Hamiltonian approach to optimal stochastic resource allocation" Adv. Appl. Prob. vol. 9. pp. 55-68, 1977.

[21] K. C. Sevcik "The use of service time distributions in scheduling," Univ. Toronto, Toronto, Canada, Tech. Rep. CSRG-14, 1972.

[22] P. Whittle. "Multi-armed bandits and the Gittins index," J. Roy. Stat. Soc. Ser. B., vol. 42 , pp. 143-149, 1980 .

\section{Feedback Decomposition of Nonlinear Control Systems}

HENK NIJMEIJER

\begin{abstract}
By using the recently developed (differential) geometric approach to nonlinear systems, a feedback decomposition for nonlinear control systems is derived:
\end{abstract}

\section{INTRODUCTION}

Consider a control system of the form

$$
\begin{aligned}
& \dot{x}=A(x)+\sum_{i=1}^{m} B_{i}(x) u_{i} \\
& z_{i}=H_{i}(x), \quad i=1, \cdots, m
\end{aligned}
$$

where $x$ are local coordinates of a smooth $n$-dimensional manifold $M, A, B_{1}, \cdots, B_{m}$ are smooth vector fields on $M$, and $H_{i}: M \rightarrow N_{i}$ is a smooth output map from $M$ to a smooth $p_{i}-\left(p_{l} \geqslant 1\right)$ dimensional manifold $N_{1}$ for $i=1, \cdots, m$. We assume that each $H_{i}, i=1, \cdots, m$, is a surjective submersion. Furthermore, we will assume that the system (1.1a) is strongly accessible (see [12]).

In this note we will study the static state feedback noninteracting control problem. That is (see [4]), we seek a control law of the form

$$
u=\alpha(x)+\beta(x) v
$$

where $\alpha: M \rightarrow \mathbb{R}^{m}, \beta: M \rightarrow \mathbb{R}^{m \times m}$ are șmooth maps, $\beta(x)=\left(\beta_{i j}(x)\right)$ is nonsingular for all $x$ in $M$, and $v=\left(v_{1}, \cdots, v_{m}\right)^{t} \in \mathbb{R}^{m}$. Let $\tilde{A}(x)=A(x)$ $+\sum_{i=1}^{m} B_{i}(x) \alpha_{i}(x)$ and $\dot{B}_{i}(x)=\sum_{j=1}^{m} B_{j}(x) \beta_{i j}(x)$. Then, in suitable local coordinates the modified dynamics $\dot{x}=\tilde{A}(x)+\sum_{i=1}^{m} \tilde{B}_{2}(x) v_{i}$ should read

$$
\left\{\left(\begin{array}{c}
\dot{x}_{1} \\
\dot{x}_{2} \\
\vdots \\
\dot{x}_{m}
\end{array}\right)=\left(\begin{array}{c}
\tilde{A}_{1}\left(x_{1}\right) \\
\tilde{A}_{2}\left(x_{2}\right) \\
\vdots \\
\tilde{A}_{m}\left(x_{m}\right)
\end{array}\right)+\left(\begin{array}{ccc}
\tilde{B}_{1}\left(x_{1}\right) & & \bigcirc \\
& \tilde{B}_{2}\left(x_{2}\right) & \\
& \ddots & \tilde{B}_{m}\left(x_{m}\right)
\end{array}\right)\left(\begin{array}{c}
v_{1} \\
\vdots \\
\dot{t}_{m}
\end{array}\right)\right.
$$

$$
\left\{\begin{array}{c}
z_{1}=H_{1}\left(x_{1}\right) \\
\vdots \\
z_{m}=H_{m}\left(x_{m}\right)
\end{array}\right.
$$

where $x=\left(x_{1}, \cdots, x_{m}\right)$ with each $x_{i}$ and $z_{i}$ possibly being a vector. For linear systems, the above problem - the restricted decoupling problem (RDP) - has been solved under the additional assumption that the set of outputs is "complete"; i.e., $\cap_{i=1}^{m} \operatorname{ker} H_{i}=0$ (see [13]). In the solution we

Manuscript received June 24. 1982.

The author was with Stichting Mathematisch Centrum, Kruislaan 413, 1098 SJ Amsterdam. The Netherlands. He is now with the Department of Applied Mathematics. Twente University of Technology, Enschede. The Netherlands. present here, we use as key tools the so-called (regular) controllability distributions introduced in [8]. In this way, our approach completely fits in the systematic work on the generalization of Wonham's geometric approach to linear systems (see, e.g., [3]-[10]). We note that a parallel decomposition as in (1.3a) has been studied in [11]. We also note that similar results are derived in [4] and in a different style in [1]. The main purpose of this note is to show that the solution of the nonlinear RDP can also be derived by directly generalizing the theory of [13].

\section{Problem Formulation}

Recall the following definitions (see [3]-[9]).

Definition 2.1: An involutive distribution $D$ of fixed dimension, on $M$, is controlled invariant for the system (1. La) if there exists a feedback of the form (1.2) such that the modified dynamics $\dot{x}=\tilde{A}(x)+\sum_{i=1}^{m} \bar{B}_{i}(x) v_{i}$ leave $D$ invariant; i.e.,

$$
\begin{aligned}
& {[\tilde{A}, D] \subset D} \\
& {\left[\tilde{B}_{i}, D\right] \subset D, \quad i=1, \cdots, m .}
\end{aligned}
$$

Definition 2.2: An involutive distribution $D$ of fixed dimension, on $M$, is a regular controllability distribution of the system (1.1a) if it is controlled invariant for the system, and moreover,

$$
D=\text { involutive closure of }\left\{a d_{\tilde{A}}^{k} \tilde{B}_{i} \mid k \in \mathbb{N}, i \in I\right\}
$$

for a certain subset $I \subset\{1, \cdots, m\}$.

Instead of the above notion of controlled invariance, it is sufficient to use a somewhat weaker concept.

Definition 2.3: An involutive distribution $D$ of fixed dimension, on $M$, is locally controlled invariant for the system (1.1a) if locally around each point $x_{0} \in M$ there exists a feedback of the form (1.2) such that the modified dynamics $\dot{x}=\tilde{A}(x)+\sum_{i=1}^{m} \tilde{B}_{i}(x) v_{i}$ leave $D$ invariant.

Similarly, one defines a local version of Definition 2.2: the regular local controllability distributions.

In considering the static state feedback noninteracting control problem, we seek regular local controllability distributions $R_{1}, \cdots, R_{m}$ defined by

$$
R_{i}:=\text { involutive closure of }\left\{a d_{A}^{k} \tilde{B}_{i} \mid k \in \mathbb{N}\right\}
$$

where $\tilde{A}$ and $\bar{B}_{i}$ are as in (1.3a), $i=1, \cdots, m$.

Remark: In the local coordinates of (1.3a) we see that $R_{i}=$ $\operatorname{span}\left\{\partial / \partial x_{i}\right\}$, and clearly each distribution $R_{i}$ satisfies $\left[\tilde{A}, R_{i}\right] \subset R_{i}$ and $\left[\tilde{B}_{j}, R_{i}\right] \subset R_{i}, j=1, \cdots, m, i=1, \cdots, m$.

Assuming (2.1), we see that

$$
R_{i} \subset \operatorname{ker} H_{j^{*}}=: K_{j} \quad j \neq i, i, j=1, \cdots, m
$$

which means exactly that $v_{j}(\cdot)$ does not affect the output $z_{i}(\cdot)$, for $j \neq i$. Second, we have the nonlinear version of output controllability; that is,

$$
H_{i^{*}}\left(R_{i}\right)=T N_{i} \quad i=1, \cdots, m
$$

This follows from the fact that the system (1.1a) is strongly accessible, so that (1.3a) is also strongly accessible. But then each of the systems $\dot{x}_{i}=A_{i}\left(x_{i}\right)+\dot{B}_{i}\left(x_{i}\right) v_{i}$ is strongly accessible, and by the fact that the map $H_{i}$ is a surjective submersion we see that the set of reachable output values has nonempty interior in $N_{i}$ for all $i=1, \ldots, m$.

Thus, the static state feedback noninteracting control problem can be stated as follows.

Given the system (1.1a) (1.1b), find (if possible) a local feedback law of the form (1.2) such that (2.2) and (2.3) hold for the distributions $R_{i}$ defined by (2.1). Now, as in the linear case, there is a compatibility problem (see [13]). Clearly, if we have controlled invariant distributions $D_{1}, \ldots, D_{m}$, then by no means does it follow that there exists a local feedback (1.2) which leaves each of them invariant. Therefore, we make the following assumption: 


$$
\bigcap_{i=1}^{m} \operatorname{ker} H_{i^{*}}=0
$$

which means that the map

$$
H: M \rightarrow N_{1} \oplus N_{2} \oplus \cdots \oplus N_{m}, \quad H(x)=\left(H_{1}(x), \cdots, H_{m}(x)\right)
$$

is locally injective.

\section{MAIN THEOREM}

Define $R_{i}^{*}:=$ supremal regular local controllability distribution in

$$
\bigcap_{j=i} \operatorname{ker} H_{j^{*}}, \quad i=1, \cdots, m \text {. }
$$

Remark: $R_{i}^{*}$ is well defined (see [6], [8]), but the dimension probably is not fixed.

Theorem 3.1: Under the assumption (2.4) and the assumption that each $R_{i}^{*}$ has fixed dimension, $i=1, \cdots, m$, the static state feedback noninteracting control problem is solvable in a local fashion if and only if

$$
R_{i}^{*}+K_{i}=T M .
$$

Proof: Assume (3.1) holds, then (2.2) and (2.3) are true for $R_{i}^{*}$. We show next that the $\hat{K}_{l}:=\cap_{j=i} \operatorname{ker} H_{j *}, i=1, \cdots, m$, are independent. Indeed,

$$
\begin{aligned}
\hat{K}_{t} \cap \sum_{j \neq i} \hat{K}_{j} & =\left(\bigcap_{r \neq i} \operatorname{ker} H_{r^{*}}\right) \cap \sum_{j \neq i}\left(\bigcap_{s \neq j} \operatorname{ker} H_{s^{*}}\right) \\
& \subset\left(\bigcap_{r \neq i} \operatorname{ker} H_{r^{*}}\right) \cap \operatorname{ker} H_{i^{*}}=\bigcap_{r=1}^{m} \operatorname{ker} H_{r^{*}}=0 .
\end{aligned}
$$

Since $R_{i}^{*} \subset \hat{K}_{i}, i=1, \cdots, m$, it follows that the $R_{i}^{*}$ are independent. In the next step, we will show that the $R_{l}^{*}$ are compatible; i.e., there is a local feedback (1.2) which leaves each of the distributions $R_{i}^{*}$ invariant. From (3.1) we see that for each $i=1, \cdots, m, R_{l}^{*} \neq 0$. For if $R_{1}^{*}=0$ for an $i \in\{l, \cdots, m\}$, then $K_{i}=T M$, which means that $z_{i}=H_{i}(x)$ is constant. Therefore, we know by the independence of the $R_{i}^{*}$ that locally there exist independent vector fields $0 \neq \bar{B}_{i}$ with $\bar{B}_{i} \in R_{i}^{*} \cap \operatorname{span}\left\langle B_{1} \cdots, B_{m}\right\rangle, i=$ $1, \cdots, m$. So, $\operatorname{span}\left\{B_{1} \cdots, B_{m}\right\}=\operatorname{span}\left(\bar{B}_{1}, \cdots, \bar{B}_{m}\right\}$. We also have that $\operatorname{dim} R_{i}^{*} \geqslant p_{i}$ (by assumption $R_{i}^{*}$ has fixed dimension), and thus from the independency of the $R_{i}^{*}$, we have $R_{1}^{*}=+\cdots+R_{m}^{*}=T M$. Thus, the distributions $R_{1}^{*}, \cdots, R_{m}^{*}$ are simultaneously integrable (see [11. Definition 3.1 and Lemma 3.1]). So locally around each point $x_{0} \in M$ there exist coordinates such that $R_{i}^{*}=\operatorname{span}\left\{\partial / \partial x_{1}\right\}, i=1, \cdots, m$, with each $x_{i}$ possibly being a vector. Now, from the fact that the distributions $R_{i}^{*}$ are locally controlled invariant we have

$$
\begin{aligned}
& {\left[\bar{B}_{1}, R_{j}^{*}\right] \subset R_{j}^{*}+\operatorname{span}\left\{\bar{B}_{1}, \cdots, \bar{B}_{m}\right\}, \quad i=1, \cdots, m} \\
& {\left[A, R_{j}^{*}\right] \subset R_{j}^{*}+\operatorname{span}\left\{\bar{B}_{1}, \cdots, \bar{B}_{m}\right\}}
\end{aligned}
$$

for all $j=1, \cdots, m$.

From (3.2a) we see that

$$
\begin{aligned}
{\left[\bar{B}_{1}, R_{2}^{*}+\cdots+R_{m}^{*}\right] } & \subset R_{2}^{*}+\cdots+R_{m}^{*}+\operatorname{span}\left\{\bar{B}_{1}, \cdots, \bar{B}_{m}\right\} \\
& =R_{2}^{*}+\cdots+R_{m}^{*}+\operatorname{span}\left\{\bar{B}_{1}\right\}
\end{aligned}
$$

where the last equality follows from the fact that $\bar{B}_{i} \in R_{i}^{*}, i=1, \cdots, m$. Note also that the distribution $R_{2}^{*}+\cdots+R_{m}^{*}$ is involutive, cf. [11]. Now, from (3.3) and [5], [7] it follows that there locally exists a vector field $\tilde{B}_{1}$ such that $\operatorname{span}\left\{\tilde{B}_{1}\right\}=\operatorname{span}\left\{\bar{B}_{1}\right\}$ and $\left[\tilde{B}_{1}, R_{2}^{*}+\cdots+R_{m}^{*}\right] \subset R_{2}^{*}+\cdots+$ $R_{m}^{*}$. Therefore, in the coordinate system constructed above we have that $\tilde{B}_{1}(x)=\left(\tilde{B}_{1}\left(x_{1}\right), 0, \cdots, 0\right)^{t}$.

Similarly, we construct vector fields $\tilde{B}_{i}, i=2, \cdots, m$, such that $\left[\bar{B}_{i}, R_{1}^{*}\right.$ $\left.+\cdots+R_{i-1}^{*}+R_{i+1}^{*}+\cdots+R_{m}^{*}\right] \subset R_{1}^{*}+\cdots+R_{i-1}^{*}+R_{i+1}^{*}+\cdots$ $+R_{m}^{*}$ and $\operatorname{span}\left\{\tilde{B}_{i}\right\}=\operatorname{span}\left\{\bar{B}_{1}\right\}$. Thus,

$$
B_{i}(x)=\left(0, \cdots, 0, B_{i}\left(x_{i}\right), 0, \cdots, 0\right)^{t} .
$$

Next, from $(3.2 b)$ we see that

$$
\left[A, R_{2}^{*}+\cdots+R_{m}^{*}\right] \subset R_{2}^{*}+\cdots+R_{m}^{*}+\operatorname{span}\left\{\vec{B}_{1}\right\}
$$

and therefore we can construct a local feedback $u=\bar{B}(x) \alpha_{1}(x)$ such that $\tilde{A}(x)=A(x)+\bar{B}_{1}(x) \alpha_{1}(x)$ satisfies (cf. [3]) $\left[\tilde{A}, R_{2}^{*}+\cdots+R_{m}^{*}\right] \subset R_{2}^{*}$ $+\cdots+R_{m}^{*}$. Similarly, for the distribution $R_{1}^{*}+\cdots+R_{i-1}^{*}+R_{i+1}^{*}$ $+\cdots+R_{m}^{*}$, we construct a feedback $u=\bar{B}_{i}(x) \alpha_{i}(x)$ such that the modified dynamics leave this distribution invariant. Finally, by applying the total feedback $u=\bar{B}_{1}(x) \alpha_{1}(x)+\cdots+\bar{B}_{m}(x) \alpha_{m}(x)$, we obtain $A(x)$ $\left.=\left(A_{1}(x)_{1}\right), A_{2}\left(x_{2}\right), \cdots, A_{m}\left(x_{m}\right)\right)$. So we have established a local feedback (1.2) such that the modified dynamics are as in (1.3a), and also from (3.1) (1.3b) is satisfied. Furthermore, we note that each system $\dot{x}_{i}=A_{i}\left(x_{i}\right)$ $+B_{i}\left(x_{i}\right) v_{1}$ is strongly accessible, and we have that

$$
R_{i}^{*}=\text { involutive closure of }\left\{a d_{\tilde{A}}^{k} \tilde{B}_{i} \mid k \in \mathbb{N}\right\}, \quad i=1, \cdots, m
$$

Conversely, from the fact that the $R_{i}^{*}$ are supremal relative to the condition (2.2) and from (2.3)-which is equivalent to $R_{i}+K_{i}=T M-$ it follows that (3.1) is necessary.

\section{REMARKS}

1) In [11, Lemma 3.1] the distributions $D_{1}, \cdots, D_{L}$ should be independent; i.e., for each disjoint subset $I_{1}$ and $I_{2}$ of $\{1, \cdots, L\}$, one has that $D^{I_{1}} \cap D^{I_{2}}=0$

2) $\left[a d_{\tilde{A}}^{k} \tilde{B}_{i}, a d_{A}^{l-\tilde{B}_{j}}\right]=0$ for all $k, l \in \mathbb{N}$ and $i=j$ (see also, [11]).

3 ) If the number of output channels is smaller than the number of inputs, the above procedure still works in a slightly modified way. Namely, there are more than one independent vector fields $\bar{B}_{i}$ in $R_{i}^{*} \cap$ $\operatorname{span}\left\{B_{1}, \cdots, B_{m}\right\}$, and/or there exist some additional input vector fields $\tilde{B}_{k}$ which do not belong to one of the distributions $R_{2}^{*}$, but-after applying feedback-also have the form $\tilde{B}_{k}(x)=\left(\tilde{B}_{k}^{1}\left(x_{1}\right), \tilde{B}_{k}^{1}\left(x_{2}\right), \cdots\right.$, $\left.\bar{B}_{k}^{m}\left(x_{m}\right)\right)^{t}$. These vector fields are superfluous for the whole control synthesis of the system.

4) Each of the systems $\dot{x}_{i}=\tilde{A}_{i}\left(x_{i}\right)+\dot{B}_{i}\left(x_{i}\right) v_{i}, z_{i}=H_{i}\left(x_{i}\right)$ is strongly invertible (see [2]). This has also been clarified in a geometric way in [9], and follows directly from the condition that $R_{i}^{*}+K_{i}=T M$, so $R_{i}^{*}$ is not contained in ker $H_{1}$. We also note that the situation described in Theorem 3.1 is even more special. Namely, the system $\dot{x}_{i}=\tilde{A}_{i}\left(x_{i}\right)+\tilde{B}_{i}\left(x_{i}\right) v_{i}$ is strongly invertible with respect to each of the components of the output $z_{i}$.

\section{REFERENCES}

[1] D. Claude. "Decoupling of nonlinear systems," Syst. Contr. Lett., vol. 1, pp. 242-248, 1982

[2] R. M. Hirschom, "Invertibility of nonlinear control systems." $S I A M J$. Contr., vol 17. pp. $289-297,1979$.

[3] - " $(A, B)$-invariant distributions and disturbance decoupling of nonlinear systems." SIAM J. Contr. Optimiz., vol. 19. pp. 1-19, 1981.

[4] A. Isidori, A. J. Krener, C. Gori-Giorgi, and S. Monaco, "Nonlinear decoupling via feedback: A differential geometric approach." IEEE Trans. Automat. Contr., vol. 26 pp. 33I-345. 1981 .

[5] -.- "Locally $(f, g)$-invariant distributions," Syst. Contr. Lett.. vol. 1, pp. 12-15,

6) A. J. Krener and A. Isidori, ( $A d f, G)$ "Invariant and controllability distributions," in Feedhack Control of Linear and Nonlinear Systems (Lecture Notes in Control and Information Sciences 39). pp. 157-164.

[7] H. Nijmeijer, "Controlled invariance for affine control sustems," Int. J. Contr., vo]. 34. pp. $825-833,1981$

[8] -_. "Controllability distributions for nonlinear systems," Syst. Contr. Lett., vol. 2 pp. 122-129, 1982.

[9] -. "Invertibility of affine nonlinear control systems: A geometric approach," Syst. Contr. Leth., vol. 2. pp. 163-168, 1982

[10] H. Nijmeijer and A. J. Van Der Schaft, "Controlled invariance for nonlinear systems." IEEE Trans. Automat. Contr., vol. 27. pp. 904-914. 1982.

[II] $\dot{w}$. Respondek, "On decomposition of nonlinear control systems," Syst. Conrr. Lett. vol. I. pp. 301-308, 1982 .

[12] H. J. Sussmann and V. Jurdjevic. "Controllability of nonlinear systems," J. Differenrial Equations, vol. 12, pp. 95-116. 1972.

[13] W. M. Wonham, Linear Mulivariable Control, A Geometric Approach, 2nd ed. New Yark: Springer-Verlag. 1979. 